\title{
Basic Readout Electronic Board for Three Channel Cosmic Ray Detector
}

\author{
Luis Arceo*t \\ Laboratorio de Partículas Elementales, División de Ciencias e Ingenierías Campus León, \\ Universidad de Guanajuato, México \\ E-mail: miquelefisica.ugto.mx

\section{Julián Félix} \\ Laboratorio de Partículas Elementales, División de Ciencias e Ingenierías Campus León, \\ Universidad de Guanajuato, México \\ E-mail: felixefisica.ugto.mx
}

\begin{abstract}
Detection of cosmic rays can be used for teaching and research. The detector and its electronics have a key role in the cosmic ray detection process. It is presented the design, construction and test of a basic readout three channel electronic board for a cosmic ray detector based on three 2.54 cm X $5.08 \mathrm{~cm}$ X $20.32 \mathrm{~cm}$ Aluminum blocks, and some very preliminary physical results.
\end{abstract}

38th International Conference on High Energy Physics

3-10 August 2016

Chicago, USA

* Speaker.

${ }^{\dagger}$ CONACYT for the support to the project 223179,10017, CB-2013-01 "Fondo Sectorial de Investigación para educación SEP- CONACYT". CONACYT for the support to the project 223179,10017, CB-2013-01 "Fondo Sectorial de Investigación para educación SEP- CONACYT". And Laboratorio de Partículas Elementales http://laboratoriodeparticulaselementales.blogspot.mx/. 


\section{Introduction}

Currently there are many reports on cosmic rays detectors [1], these are with different geometric configurations, and based on different physical detection processes and electronic boards. This paper focuses on the design, construction and test of a basic readout electronic board for a cosmic ray detector, based on the Hamamatsu photodiode S12572-100P [2] and a $2.54 \mathrm{~cm} \mathrm{X} 5.08$ $\mathrm{cm}$ X $20.32 \mathrm{~cm}$ Aluminum block. We present the design, construction, and preliminary results of the electronic board and cosmic ray detector.

\section{Design and Construction}

The design of the cosmic rays detector contains five electronic boards as follows: 1) Photodiode board, its function is to support mechanically the electrical connection of the photodiode to attach it to the Aluminum block on one of its polished $2.54 \mathrm{~cm} \mathrm{X} 5.08 \mathrm{~cm}$ end. 2) Passive electronic board, is the base of the RC circuit to decouple the input high voltage from the low output voltage. 3) RF transformer board, its function is to couple the impedance between passive electronic board and amplifier board [3]. 4) Amplifier board, its function is to amplify the analogue signal by two [4]. 5) Discriminator board, its function is to compare the analogue signal, coming from the amplifier board, with a fixed trigger voltage defined by the final user and give out the digital signal [5].

The previous output signal was stored with the compactRIO -cRIO- NI-9026 [6] and NI-9402 digital input/output port by National Instrument. The program reads the digital input signal every $25 \mathrm{~ns}$ and send the counts information to the PC-Host every $1 \mathrm{~ms}$. In picture 1 is displayed the block diagram, above explained, for one channel cosmic ray detector. The complete cosmic ray detector has three channels in stack arrangement.

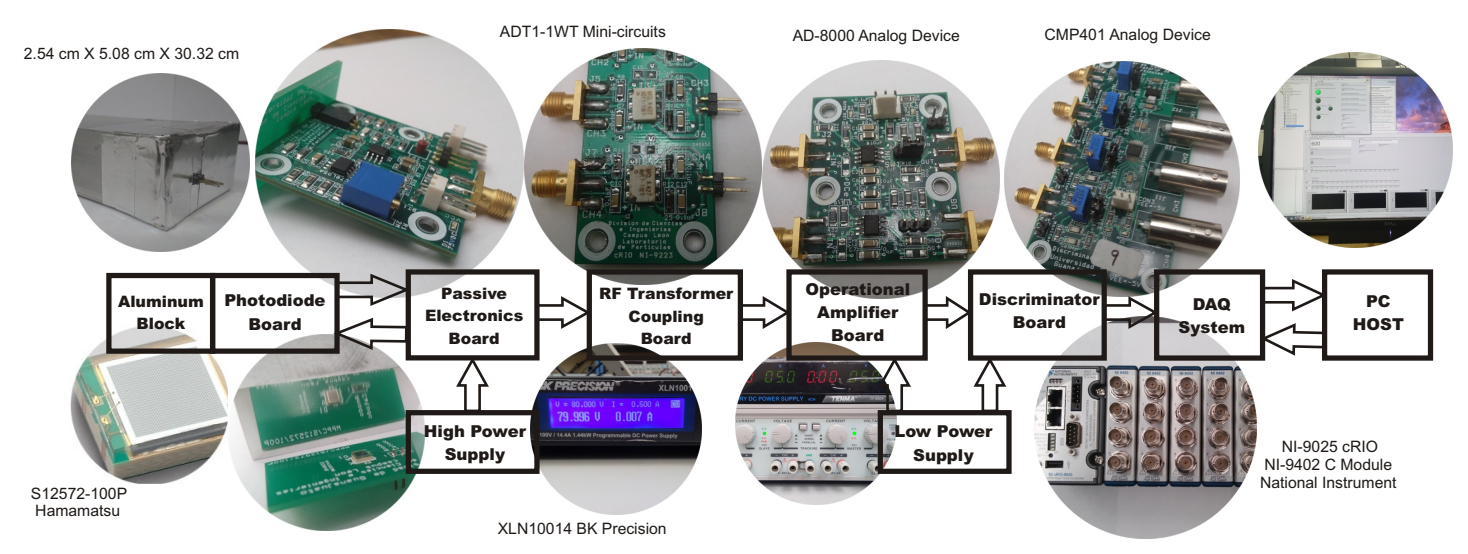

Figure 1: Block diagram of one-channel of cosmic ray detector.

\section{Characterization}

We studied the output counts as function of applied high voltage for each channel. The recording interval began at 60 volts and finished at 100 volts in steps of 5 volts each. Nine ten minutes 
text files were generated. Figure 2 corresponds to the results of top Aluminum block. Figure 3 corresponds to the result of middle Aluminum block. And figure 4 corresponds to the result of bottom Aluminum block. In the three channels for voltages between 60 and 65 volts the result is zero counts. For 70 volts and higher the number the counts increases lineally with applied voltage.

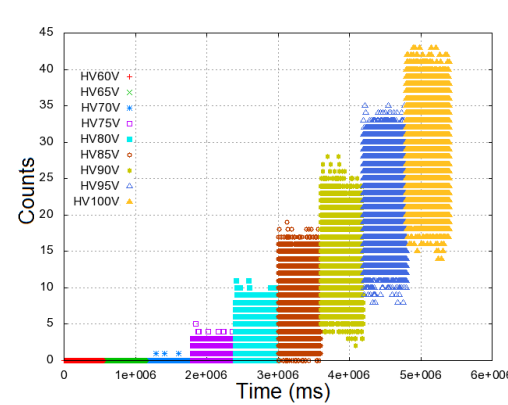

Figure 2: Top Aluminum block.

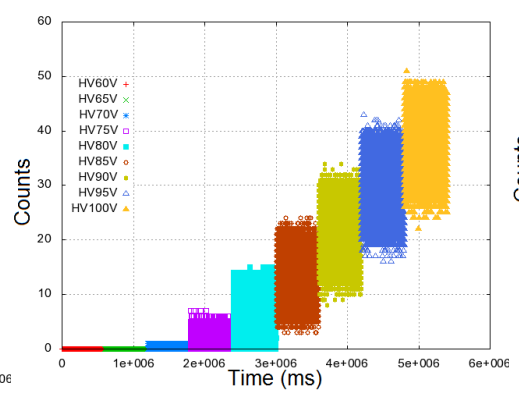

Figure 3: Middle Aluminum block.

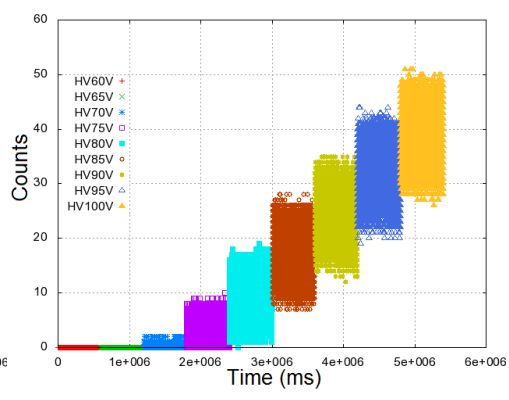

Figure 4: Bottom Aluminum block.

\section{Results}

The analogue signal noise was $20 \mathrm{mVpp}$ and the configuration parameters were $100 \mathrm{~V}$ for operation voltage and $100 \mathrm{mV}$ of threshold for the discriminator board. The figure 5, 7 and 9 display the number of counts vs time for top, middle and bottom channels, respectively. The figure 6,8 and 10 display frequency vs counts histograms for top, middle and bottom channels, respectively. The data was recorded at $\mathrm{N} 41^{\circ} 51^{\prime} 19.3$ " and W $88^{\circ} 14^{\prime} 34.33^{\prime \prime}$ at 216 m.a.s.l.

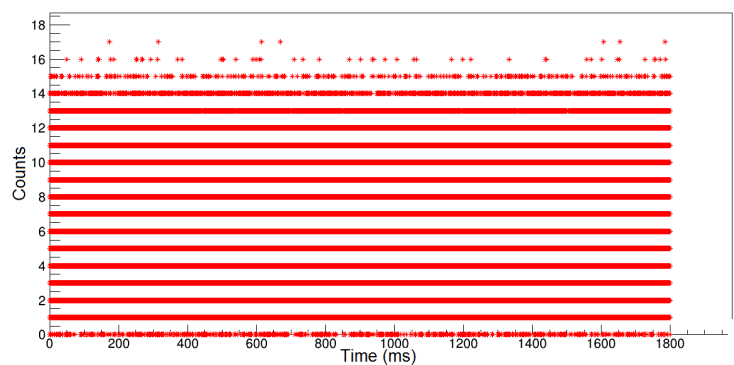

Figure 5: Counts vs time of top Aluminum block.

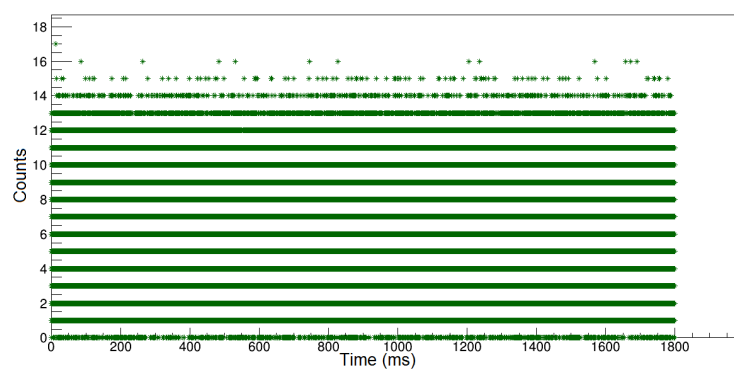

Figure 7: Counts vs time of middle Aluminum block.

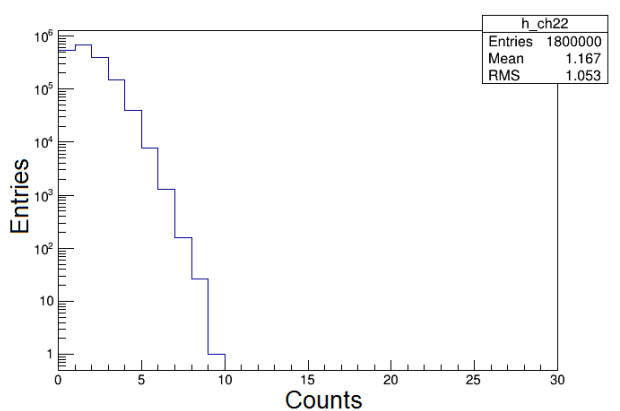

Figure 6: Frequency vs counts of top Aluminum block.

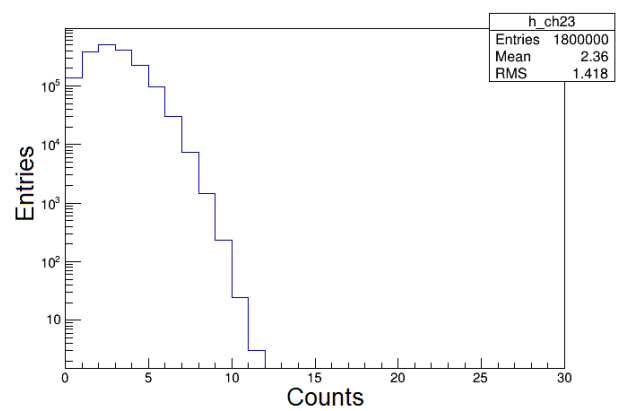

Figure 8: Frequency vs counts of middle Aluminum block. 


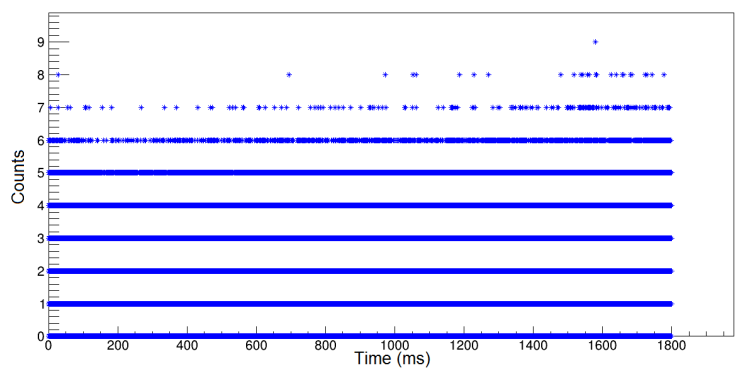

Figure 9: Counts vs time of bottom Aluminum block.

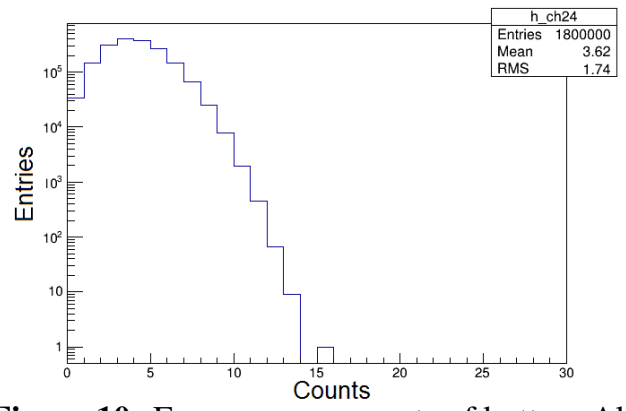

Figure 10: Frequency vs counts of bottom Aluminum block.

\section{Conclusions}

We have designed, constructed and tested a three channel cosmic ray detector. The electronic board, the DAQ System and photodiode work properly. We have characterized the cosmic ray detector to obtain a linear function of the counts vs applied high voltage. The distributions of counts vs time are almost flat, for the three channels of the cosmic ray detector. The distributions of frequency vs counts are almost a Poisson distribution, for the three channels of the cosmic ray detector.

\section{References}

[1] http://inspirehep.net/record/912798/files/CRA\%20School_027.pdf.

[2] Hamamatsu, datasheet, (December, 2015). http://www.hamamatsu.com/jp/en/S12572-100P.html.

[3] Mini-Circuits, datasheet, (Febrary 25, 2011). https://www.minicircuits.com/pdfs/ADT1-1WT.pdf.

[4] Analog Devices, datasheet, (Rev. C, 2015-2016).

http://www.analog.com/media/en/technical-documentation/data-sheets/AD8000.pdf.

[5] Analog Devices, datasheet, (Rev. A, 2002).

http://www.analog.com/media/en/technical-documentation/data-sheets/CMP401_402.pdf.

[6] National Instruments, user manual and specifications NI cRIO-9025 (October, 2015). Http://www.ni.com/pdf/manuals/375490d.pdf. 